\title{
Performance Prediction Modeling of Concrete Bridge Deck Condition Using an Optimized Approach
}

\author{
Aqeed Mohsin Chyad ${ }^{1}$, Osama Abudayyeh ${ }^{2}$ \\ 1. Dept. of Civil and Construction Engineering, Training and Energy Researches Office, Ministry of Electricity, \\ Baghdad, Iraq \\ 2. Dept. of Civil and Construction Engineering, Western Michigan Univ., Kalamazoo, MI 49008, USA \\ E-mail: aqeedmohsinch.chyad@wmich.edu (Corresponding author); Osama.abudayyeh@wmich.edu
}

Received: 25 March 2020; Accepted: 1 June 2020; Available online: 30 June 2020

\begin{abstract}
Developing an accurate and reliable model for concrete bridge deck deterioration rates is a significant step in improving the condition assessment process. The main goal of this study is to develop a deterioration prediction model based on the condition ratings of concrete bridge decks over the past 25 years as reported in the National Bridge Inventory (NBI) database. While the literatures have typically suggested the Markov chain method as the most common technique used in condition assessment of bridges, the analysis in this pilot study suggests that the lognormal distribution function is a better model for concrete bridge deck condition data. This paper compares the two approaches and presents a new approach that combines the more commonly used Markov chain method with the lognormal distribution function to arrive at an optimal model for predicting bridge deck deterioration rates. The prediction error in the combined model is less than each of the two models (i.e. Markov and Lognormal). Additionally, the steel structure type illustrated the highest deterioration rates within condition ratings from 8 to 4 Comparing with other types. The bridge decks that have ADT of more than 4,000 (vehicles/day) deteriorated faster than of those with ADT less than 4,000 with the same type of structure and skew angle. Bridge decks with skew angles more than $30^{\circ}$ deteriorate faster than of those with skew angles less than $30^{\circ}$. Furthermore, it showed that most new Michigan concrete bridge decks may take at least 40 years before dropping gradually from 9 to 3.
\end{abstract}

Keywords: Time in Condition Rating (TICR); Condition Ratings (CRs); Average Daily Traffic (ADT); National Bridge Inventory (NBI); Federal Highway Administration (FHWA).

\section{Introduction}

Maintaining the safety and serviceability of concrete bridge decks is crucial because bridges are a critical part of the transportation network. For this reason, the condition of a bridge must be evaluated periodically [1] as it is often difficult to completely reconstruct or rehabilitate a bridge that is either structurally deficient or functionally obsolete because of limited funds. Therefore, the primary goal of a bridge management system (BMS) is to allow transportation agencies to predict the future condition of bridge elements [2]. Typically, BMS uses accurate and reliable prediction models to compute the deterioration rates of the various bridge elements, which are then used in making maintenance, rehabilitation, or replacement decisions to minimize expenditures [3,4]. Several prediction methods have been developed since the early 1970s to measure deterioration rates of bridges using bridge inspection data. These methods can be used to optimize the distribution of scarce capital resources on maintenance, repair, and rehabilitation actions. The Federal Highway Administration (FHWA) has developed the National Bridge Inventory (NBI) database as a main source of data for transportation agencies to use in analyzing and predicting the condition of a bridge [5,6].

Remaining service life, time of maintenance, and effect of external factors on the condition state of concrete bridge decks can be estimated from the deterioration rates, which can be calculated by mathematical methods [7, 8]. Several methods have been explored as models for predicting the condition of bridges, including the fuzzy based analytic hierarchy approach [1,9], deterministic methods [10,11], probability distribution methods [12,13], and Markov chain models [3,14]. While deterioration rates and predictions of the future condition of bridges can be determined using these methods, each method has its advantages and limitations based on the data observed.

Some researchers have studied the service life of bridges. Twenty-eight bridges were tested to evaluate the average life anticipation for the first time. Eighty years were concluded to be the average life of a bridge. In these datasets, the main components (Superstructures, Substructures, Foundations) were deteriorated more than other components in the bridges [11]. The expected condition rating at a certain time and the probability of failure to 
drop from the good condition to poor condition was calculated using the estimated transition matrix. It was concluded that the estimated transition probability model was a sound and robust method used to measure bridge deterioration according to transportation network agency rules [3]. A different study estimated inspection intervals for bridges using statistical analysis. This study showed that the Weibull distribution was likely the best fit for Oregon data. The author showed that bridges with a good condition rating according to the National Bridge Inspection Standard (NBIS), tend to stay in that rating longer than 2 years. This study also showed that the time in condition ratings (TICRs) for bridges in fair to poor condition is shorter than for bridges in good condition [12].

The main goal of this study is to improve the condition assessment process for concrete bridge decks by developing an optimum deterioration prediction model that is based on the NBI condition rating data in the past 25 years (1992 to 2016). The investigation will mainly focus on Michigan data as a case study for developing and evaluating the optimum model. Additionally, the factors affecting deck deterioration will be investigated to determine their impact on the performance of concrete bridge decks over time.

\section{Problem statement}

Keeping bridges in good condition during their service lives continues to be a challenge [15]. The limited resources, restricted funds, and inadequate condition evaluations are the main difficulties in the life cycle of bridges. These issues will cause poor planning for bridge maintenance, rehabilitation, and reconstruction actions. Consequently, durability problems and poor performance levels of concrete bridge decks can result. Additionally, the condition assessment of concrete bridge decks is complicated as it is influenced by several factors including environmental, average daily traffic (ADT), age, deck area, main structure type, material type, and design loads [16]. Most of these factors are expected to have significant effects on deck deterioration. Therefore, transportation agencies have been dealing with deterioration challenges by attempting to establish optimal guidelines to maintain bridges in good condition [17]. The NBI database was developed by the FWHA to be a dependable reference for engineers to analyze and predict the condition of a bridge [6].

Although there are few studies in the literature that have focused on the deterioration rates of overall bridge structures, evaluating and predicting the condition of individual bridge elements is still lacking. There are no studies that have focused on a comprehensive evaluation of the condition of bridge decks (one key element of a bridge) using the entire 25-year period of the NBI database. Furthermore, exploring the reasons behind the deterioration of key bridge elements such as the deck is critical and needed, but is also still lacking in the literature. Consequently, understanding the process of concrete bridge deck deterioration and evaluating the condition in an accurate and reliable manner will help in better planning for the allocation of funds for bridge maintenance, rehabilitation, and reconstruction actions. Additionally, studying the various factors that have an impact on concrete bridge deck deterioration will enhance the accuracy of the evaluation of the current and future condition, resulting in improvements in the condition assessment of bridge decks and in optimizing the allocation of the limited resources and constrained funds.

\section{Condition ratings for concrete bridge decks}

The NBI database contains condition ratings for all main components of bridges which are collected by states during regular inspection intervals which are performed yearly or biennially $[13,18]$. Since bridge decks provide the riding surface and are directly in contact with traffic loads, they are more prone to deterioration than other bridge elements. Additionally, the condition assessment of concrete bridge decks is complicated and is influenced by several factors including the environment, ADT, main structure type, material type, and skew angles [11,13]. Some of these factors have significant impact on deck deterioration [11]. Therefore, the deck is considered one of the most important elements of a bridge $[10,19]$. Routine maintenance, rehabilitation, or replacement actions will be required as bridge decks deteriorate with time.

Condition ratings vary from 0 to 9 according to the NBI database [20]. Typically, bridges are considered structurally and functionally deficient if they are rated as 3 or less, and are fully functional if they receive a condition rating of 7 or more. While the American Association of State and Highway and Transportation Officials (AASHTO) has recently changed the 0 to 9 rating system to the new 1 to 4 condition states, the FHWA has not yet adopted the new system and still uses the 0 to 9 ratings in the NBI database [21]. The condition ratings for all major components of bridges are rated 9 when they are newly constructed. They stay at the same condition rating for a period of time and then start to deteriorate and drop to the next lower rating level [10]. Since condition ratings of 3 or less are considered critical and immediate action such as rehabilitation or reconstruction are necessary, researchers do not include these condition ratings when developing prediction models [12]. At these condition states, transportation agencies will essentially be looking for the necessary resources to immediately rectify the problems. 


\section{Predictive modeling}

Developing a deterioration prediction model that is based on the condition ratings of a bridge is an important step for better understanding its performance level. Additionally, to determine an accurate allocation of funds for needed maintenance, rehabilitation, or replacement actions, information on current and future bridge deck conditions are needed. Data on the current condition ratings of bridges can be obtained from the NBI database. However, to accurately evaluate the deterioration rates, inspection data must be treated to eliminate the effects of issues other than maintenance on the increase or decrease in the condition ratings and to remove errors in the data.

\subsection{Data filtration and assumptions}

Inspection data are available since the 1992 for all major bridge elements, creating a 25-year database for analysis and modeling. In the pilot study described in this paper, the focus is on Michigan concrete bridge decks inspection data which have been arranged in descending order from the highest to the lowest ratings starting from the year 1992 through 2016. Inappropriate or incomplete records are removed from the original data to achieve an accurate and reliable prediction of bridge deck deterioration rates. For example, since there was no information about the condition ratings before 1992 and after 2016, the number of years used as threshold at the beginning and end of the 25 years should be determined to get robust results. Sensitivity analysis was performed to calculate the best threshold based on different possible clipping values ranging from 3 to 7 years [12]. The results showed that 3 years is the most conservative and appropriate clipping threshold that would be used at the beginning and end of the data records. For example, if the bridge was in the condition rating of 7 from 1992 to 1993 (i.e. 2 years) and then dropped to a condition rating of 6 or jumped to a condition rating of 8 due to rehabilitation or repair in 1994, then the data from 1992 to 1993 were clipped. Similarly, if the condition rating of the bridge changed to 6 in 2015 and stayed at 6 through 2016, this data was also clipped.

Some of the condition rating data of concrete bridge decks displayed an increase or decrease in the rating for about 1 or 2 years, and then returned to its rating before dropping without any maintenance, repair, and/or replacement records. This situation can be interpreted as an inspector error, and the condition ratings are manually revised to match the previous and next condition states. For example, if the concrete bridge deck was at condition rating of 8 for 4 years, then dropped to a condition rating of 7 for 2 years, and then returned to the original value of 8 for 3 years, then this would be considered an error and would be corrected to display the concrete bridge deck at condition of 8 for 9 years.

\subsection{Concrete bridge deck statistical predictive models}

The most common stochastic statistical methods used for predicting the condition of bridges are the exponential, Weibull, lognormal, normal, and gamma distributions [12,22]. In this study, the condition rating data for concrete bridge decks were modeled using these statistical distribution functions and the best method was chosen based on the Anderson-Darling test, which is the most appropriate goodness-of-fit method for these types of data and functions [23,24].

In this pilot study, the condition ratings of Michigan concrete bridge decks ranging from 9 to 4 over the past 25 years (1992 to 2016) were used for the development of the statistical predictive models using the distribution functions (exponential, Weibull, lognormal, normal, and gamma distributions). Table 1 shows the AndersonDarling test values of the five statistical methods used in this study, which are calculated for each condition rating (9 to 4). Based on these results, the lognormal probability distribution function seems to be the best fit for Michigan concrete bridge deck condition ratings. It is also clear from Table 1 that the lognormal distribution method is the most desirable model with the smallest Anderson-Darling test values. Therefore, the lognormal distribution function was used to develop the deterioration prediction curves for Michigan concrete bridge decks.

Table 1. Anderson-Darling Test Values of Michigan Concrete Bridge Decks

\begin{tabular}{ccccccc}
\hline CR & Normal & Gamma & Lognormal & Weibull & Exponential & \# of Samples \\
\hline 9 & 8.7 & 5.4 & 4.9 & 9.3 & 38.2 & 189 \\
8 & 31.1 & 25.7 & 25.3 & 23 & 200.1 & 1837 \\
7 & 125.7 & 54 & 45.3 & 102.2 & 374.5 & 3696 \\
6 & 39.5 & 24.7 & 24.5 & 33.3 & 255.8 & 2004 \\
5 & 21.2 & 10.1 & 10.5 & 18.5 & 91.6 & 699 \\
4 & 6.5 & 3.3 & 2.9 & 6.3 & 34.6 & 199 \\
\hline
\end{tabular}

The probability density function of the lognormal distribution is denoted by [25]:

$$
f(t ; \mu, \sigma)=\frac{1}{t \sigma \sqrt{2 \pi}} \exp \left\{-\frac{(\ln t-\mu)}{2 \sigma^{2}}\right\}, \mathrm{t}>0,-\infty<\mu<\infty, \sigma>0
$$


If $t$ is a random variable that has a log-normal probability distribution, then $Y=\ln (t)$ is normally distributed with scale parameter $\mu$ and the shape parameter $\sigma$.

Where, $f(t ; \mu, \sigma)$ is the probability density function of the lognormal distribution; $t$ is independent positive random variable; $\sigma$ is the shape parameter and can be calculated as

$$
\sigma=\sqrt{\frac{\sum_{i=1}^{N}\left(\ln \left(t_{i}\right)-\mu\right)^{2}}{N}}
$$

$e^{\mu}$ is the scale parameter where $\mu$ can be calculated as

$$
\mu=\frac{\sum_{i=1}^{N} \ln \left(t_{i}\right)}{N}
$$

$N$ is Number of Samples; $i$ is the condition rating of a bridge deck $(9,8, \ldots ., 4)$; $m$ is the mean and can be calculated as

$$
\mathrm{m}=e^{\mu+\sigma^{2} / 2}
$$

$\mathrm{V}$ is the variance and can be calculated as:

$$
\mathrm{V}=\left(e^{\sigma^{2}}-1\right) e^{2 \mu+\sigma^{2}}
$$

$\mathrm{S}$ is the standard deviation and can be calculated from

$$
\mathrm{V}=\mathrm{S}^{2}
$$

In this study, $t$ is a random variable that represents the time in condition rating (TICR) in years that a bridge deck stays at a particular condition rating. For example, if a bridge deck remained in a condition rating of 8 for a period of ten years, then $t$ will be 10 . The shape parameter $(\sigma)$ is always more than 0 . It has a positive relationship with the skewness of the lognormal distribution. An increase in the value of $\sigma$ means an increase in skewness of the distribution and vice versa, where skewness represents the symmetry of the distribution. Additionally, when the value of $\sigma$ becomes less than 1 , the lognormal distribution becomes very close to the normal distribution model. The scale parameter $e^{\mu}$ indicates the average of TICR for the concrete bridge deck.

\subsection{Markov chain modeling}

The Markov chain method is also one of the most commonly used approach for modeling and predicting bridge performance levels $[7,26]$. This method is based on the concept of component conditions transitioning from one state to another during a given amount of time. The transition probability matrix is a key in using the Markov chain method because it is the basis for predicting the condition of the component $[7,27]$. This transition probability matrix is commonly attained using statistical data of bridge conditions. The transition probability matrix of a future state depends only on the current state. In this process, both time and state are discrete parameters. In the current study, the condition ratings of Michigan concrete bridge decks were modeled using the Markov chain method for six condition ratings (i.e., 9 to 4). Each condition rating can be defined according to the Markov process as a state. For example, state 1 represents a condition rating of 9, state 2 indicates a rating of 8 , and so on. The probability of a bridge component transforming from a certain condition rating to the next rating during one transition time is referred to as $\mathrm{P}_{\mathrm{ij}}$. The Markov chain transition probabilities for the 9 to 4 condition ratings of Michigan concrete bridge decks are represented by the matrix $\mathrm{P}$ as follows:

$$
\mathrm{P}=\left[\begin{array}{llllll}
P 11 & P 12 & P 13 & P 14 & P 15 & P 16 \\
P 21 & P 22 & P 23 & P 24 & P 25 & P 26 \\
P 31 & P 32 & P 33 & P 34 & P 35 & P 36 \\
P 41 & P 42 & P 43 & P 44 & P 45 & P 46 \\
P 51 & P 52 & P 53 & P 54 & P 55 & P 56 \\
P 61 & P 62 & P 63 & P 64 & P 65 & P 66
\end{array}\right]
$$

Since it is assumed that the deterioration of a bridge component can drop just one step, the probability of a bridge element staying at the same rating during a given period of time can be defined as $1-\mathrm{P}_{\mathrm{ij}}$., and the probability to transform more than one rating is considered zero. Therefore, unless there are repair or rehabilitation records, 
the bridge component condition either remains at the same rating or transforms from a particular condition rating to the next lower rating during one transition time and the probability of transforming from a low to a high rating is considered zero.

Essentially, the condition rating of a bridge component decreases over time. However, some bridge decks may have the same condition rating while at different ages. Therefore, bridge deck ages were classified into groups so that at each group the Markov chain was assumed to be homogeneous [28]. Each group consisted of 5 years and had its own transition probability matrix, which was different from the other groups. If the current or initial condition of the bridge deck is known, the future condition after a period of time (t) can be calculated by multiplication of the initial-condition vector and the transition probability matrix.

$$
C_{v}(t)=C i(0) * P^{t}
$$

Where, $C_{v}(t)=$ condition vector at any time $t ; C_{i}(0)=$ initial condition of deck $(\mathrm{t}=0) ; P^{t}=$ the transition probability matrix $\mathrm{P}$ raised to the power $\mathrm{t}$.

The most common challenge in the Markov chain method is calculating the transition probability matrix [28]. Although updating this method with new data is difficult, it is considered appropriate for this type of analysis. In this study, the percentage method was used because it needs at least two consecutive condition ratings. The initialcondition vector for bridges that are newly built is $\left[\begin{array}{llllll}1 & 0 & 0 & 0 & 0 & 0\end{array}\right]$, while for bridges that are in a rating of 7 , for example, the initial condition rating can be defined as $\left[\begin{array}{llllll}0 & 0 & 1 & 0 & 0 & 0\end{array}\right]$, and so on.

\section{Nonlinear regression predictive model}

While it has been observed from this study that the lognormal and Markov chain bridge deck condition prediction models are comparable, the authors attempted to develop an optimized approach by developing a nonlinear regression model that combines the lognormal and Markov chain models. In this approach, the best fit nonlinear regression curve is created for the data generated from the lognormal and the Markov chain models. A combination between the best prediction of each model and for each condition rating is developed. For example, if the lognormal method is the best prediction in condition rating of 9 and the Markov chain model is the best fit in condition rating of 8 , then the combination can be created between the results of lognormal and Markov chain methods at condition rating of 9 and 8, respectively. Therefore, evaluating each model for predicting the condition rating of concrete bridge deck is necessary. This evaluation is accomplished by calculating the error rates for the results of each model.

The condition rating of Michigan concrete bridge decks can be expressed by the following nonlinear regression model [29]:

$$
C R(t)=\beta_{1}+\beta_{2} t+\beta_{3} t^{2}+\beta_{4} t^{3}+\beta_{5} t^{4}
$$

Where, $C R(t)=$ The condition rating of bridge deck at any time of service; $t=$ Bridge deck age or number of years since last major reconstruction; $\beta 1$ to $\beta 5=$ Coefficients to be determined.

The coefficients will be obtained and discussed later based on the results of both lognormal and Markov chain models.

\section{Result and discussion}

In this section, the evaluation of the three predictive modeling approaches for concrete bridge decks will be presented and discussed.

\subsection{Comparative analysis of the condition assessment models}

The lognormal distribution function has been found to be the best probability distribution method to fit the condition ratings of Michigan concrete bridge decks. To assess whether the lognormal method is comparable to the Markov chain model in predicting the condition of bridge decks, the deterioration curves of Michigan bridge decks were developed using both approaches. It was concluded that lognormal results are close to those derived from the Markov chain method, see Fig. 1. Both curves are typically S-shaped, suggesting that the deterioration is fast at the beginning and end, and slow in the middle. The results illustrate that Michigan concrete bridge decks will take 16 years to drop from a condition rating of 9 to 7 (the good range) under the Markov chain method and 21 years under the lognormal model. Additionally, bridge decks will take 11 years to drop from 6 to 4 (fair range) under the lognormal model and 11 years under the Markov chain method. Moreover, the curves show that it will take 42 years for bridge decks to deteriorate from a condition rating of 9 to 4 with the lognormal model, and 40 years with the Markov chain method. These results demonstrate that the two methods are comparable. Therefore, 
the lognormal method is considered a new approach in predicting the condition ratings of bridge decks compared to the existing Markov chain method. In other words, the deterioration rates in both methods are around 0.2 $\mathrm{CR} /$ year at the beginning and end of the curves, while in the middle they are around $0.1 \mathrm{CR} /$ year.

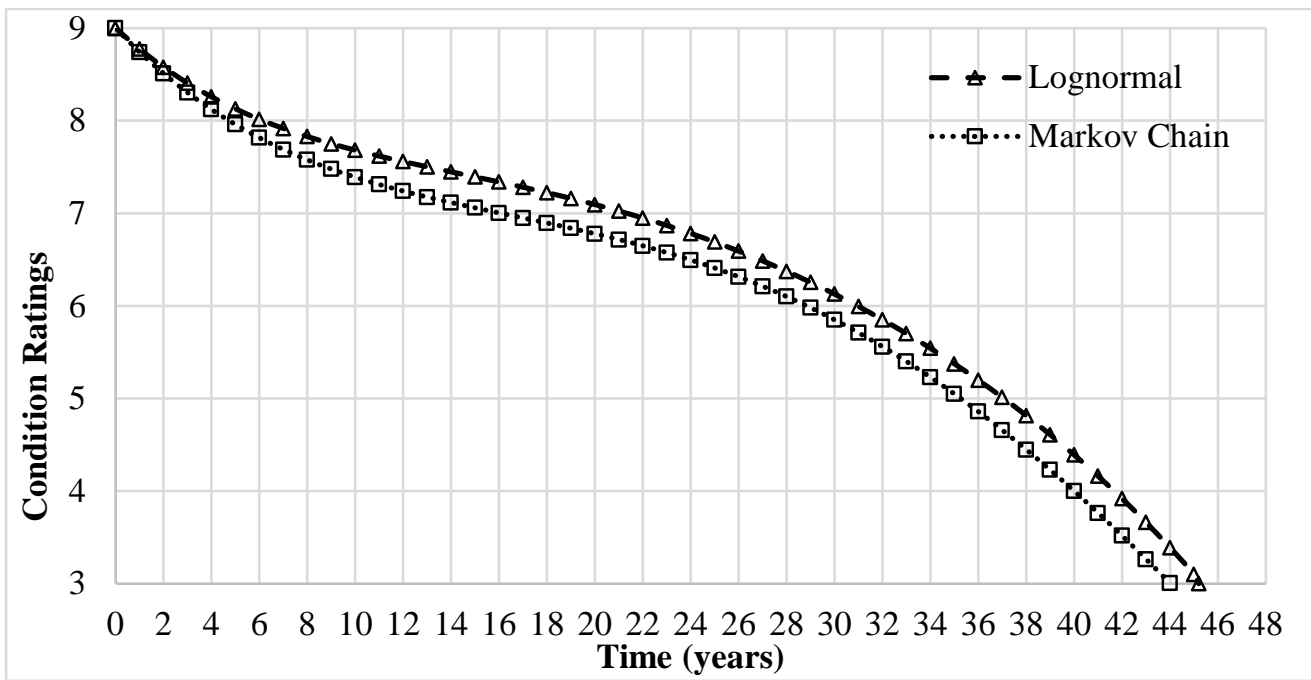

Fig. 1. Deterioration Curves of Michigan Concrete Bridge Decks Using Lognormal, Markov Chain Methods.

To further evaluate the results obtained from the lognormal and Markov chain models, error rates were computed based on the deviation of the prediction results from the original data represented in the condition ratings of concrete bridge decks. The error rates under the two methods are displayed in Table 2.

Table 2. Error Rates of Lognormal and Markov Chain Models.

\begin{tabular}{ccccc}
\hline \multirow{2}{*}{ Condition } & \multirow{2}{*}{ CR } & \multicolumn{3}{c}{ Error Percentage (\%) } \\
\cline { 3 - 5 } & & Lognormal & Markov & Nonlinear Regression \\
\hline \multirow{2}{*}{ Good Condition } & 9 & 4.74 & 5.59 & 4.73 \\
& 8 & 3.91 & 5.53 & 5.45 \\
Fair Condition & 7 & 7.10 & 5.26 & 5.6 \\
\hline & 5 & 10.58 & 7.97 & 7.54 \\
& 5 & 11.96 & 9.62 & 11.99 \\
\hline
\end{tabular}

As demonstrated by Table 2, the lognormal model had the smallest error rates for the condition ratings of 9 and 8, while the Markov chain method showed the smallest error rates for condition ratings 7, 6, 5, and 4. The TICR for all Michigan concrete bridge decks and for each condition rating from 9 to 4 have been taken into account in this calculation. Basically, the condition rating calculated by each method (lognormal and Markov chain) was compared with the original data to find the error rates. In this calculation, the minimum and maximum values of TICR for each condition rating have been taken into consideration. For example, if the minimum and maximum values of TICR for condition rating of 9 were 3 and 8 years respectively, then the original data can be arranged in the following manner $\{9,9,9,9,9,9,9,9 ; 9,9,9,9,9,9,9,8 ; 9,9,9,9,9,9,8,8 ; 9,9,9,9,9,8,8,8 ; 9,9,9$, $9,8,8,8,8 ; 9,9,9,8,8,8,8,8\}$. Now consider the TICR for condition rating of 9 was 6 years, then the original data would be $\{9,9,9,9,9,9,8,8\}$ and the lognormal CR values would be $\{8.999,8.7744,8.5783,8.4073,8.2584$, 8.1288, 8.0156, 7.9166 based on the deterioration curve of the lognormal model. The error rate is then calculated as $\{(9-8.999) / 9+(9-8.7744) / 9+(9-8.5783) / 9+(9-8.4073) / 9+(9-8.2584) / 9+(9-8.1288) / 9\} * 100 / 6=5.28 \%$. This approach is then repeated for all TICR values at CR of 9 to take the average error rate. Similarly, error values were calculated for the remaining CRs (i.e. 8, 7, 6, 5, and 4). It is worth noting that for CR of 8, the error rates are calculated starting from CR of 8 to CR of 7, and the same applies for all remaining CRs.

\subsection{Evaluation of the nonlinear regression model}

In this section, the best fit nonlinear regression curve was created for the data generated from the lognormal model in the good condition range (9 and 8) and from the Markov chain model for the condition range from 7 to 4. These range selections were based on the minimum error rates for the lognormal and Markov chain models as shown in Table 2. Therefore, the coefficients in equation (9) were obtained based on the final results of lognormal 
at condition ratings of 9 and 8 and Markov chain at condition ratings of 7 to 4 . Consequently, the best fit nonlinear regression model for Michigan data can be expressed as:

$$
C R(t)=9.175-0.285 t+0.01485 t^{2}-0.000395 t^{3}+3 * 10^{-6} t^{4}
$$

Fig. 2 shows the deterioration rates of concrete bridge decks using the nonlinear regression model based on the equation (10). It is observed that the trend of the nonlinear regression curve follows the same behavior of both the lognormal and Markov chain models. To evaluate the results obtained from the nonlinear regression modeling approach, error rates were computed based on the deviation of the prediction results from the original data represented in the condition ratings of concrete bridge decks using the same error calculations approach. The error rates under the nonlinear regression modeling approach are displayed in Table 2.

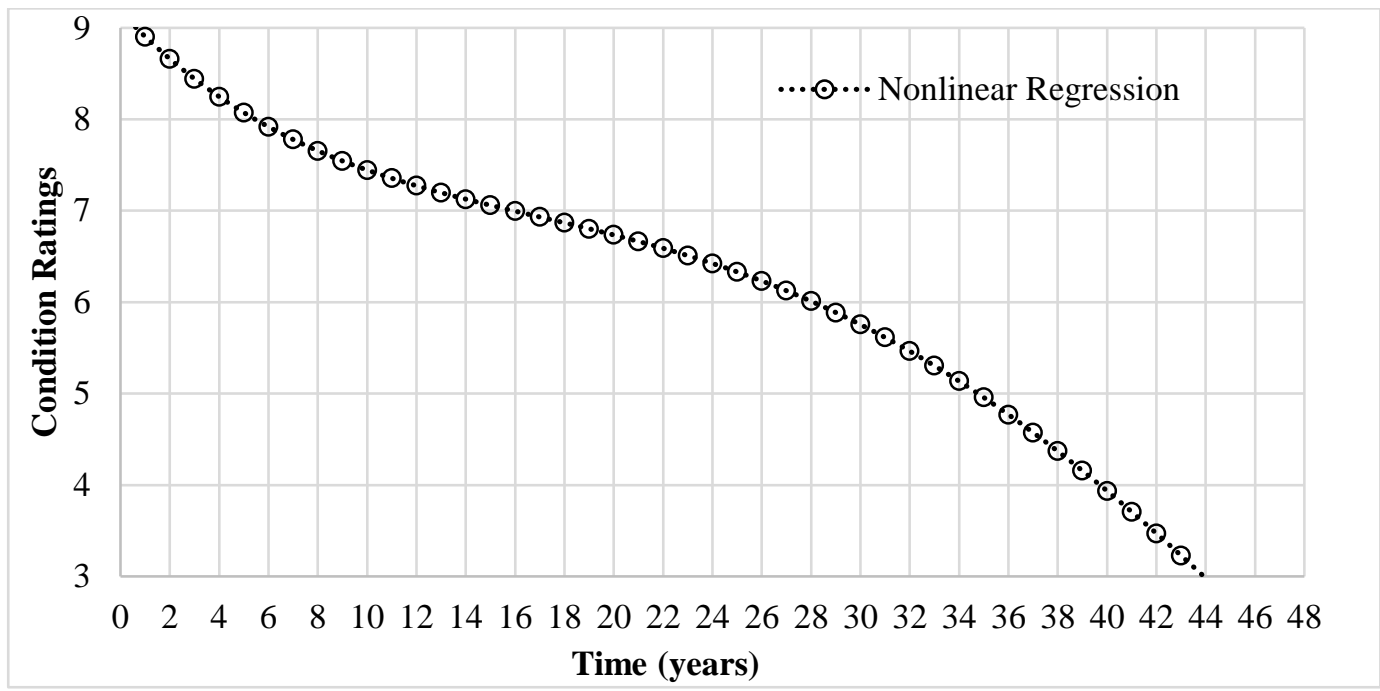

Fig. 2. Deterioration Curve of Michigan Concrete Bridge Decks Using the Nonlinear Regression Model

As shown in Table 2, the nonlinear regression model had the smallest error rates for the condition ratings of 9, 6, 5, and 4. while for the condition rating of 8 and 7, the lognormal and Markov chain methods showed the smallest error rates respectively (Table 2). Additionally, the average error rates for all condition ratings from 9 to 4 are 7.37, 7.83, and 9.38 for nonlinear regression, Markov chain and lognormal models respectively. These results showed that the nonlinear regression model had the smallest average error rates.

\section{Analysis of the factors impacting bridge deck condition}

There are several factors that may have an effect on the deterioration rates of bridges such as ADT, skew angle, environmental factors, structure type, material type, number of spans, the number of traffic lanes, and deck width $[10,11]$. In this pilot study, the ADT, structure type, and skew angle factors were selected to investigate their impact on the concrete bridge deck deterioration to demonstrate how the nonlinear regression model can be used for predicting bridge deck performance. To study the effect of these factors on the deck condition over time, each factor was considered independently from the impact of all others. This was accomplished by identifying the appropriate ranges for a given factor while keeping all others constant. The following is a summary of the factor ranges in this study:

1) The ADT factor (Item 29 in NBI data) which are divided into two categories: less than or equal to 4,000 and more than 4,000 vehicles per day.

2) The SA factor (Item 34 in NBI data) which are classified into two groups: less than or equal to $30^{\circ}$ and more than $30^{\circ}$.

3) The main structure type (Item 43 in NBI data). There are three main superstructure types that are used: concrete, prestress, and steel.

\subsection{The superstructure type factor}

Concrete, steel, and prestress are considered the main structure types for most U.S. bridges, and specifically those in Michigan that are currently in service. Therefore, knowing the bridge behavior under the effect of each type of structure may be useful in deciding how to distribute resources for bridge deck maintenance, rehabilitation, and replacement actions. Figure 3 shows the deterioration curves of Michigan concrete bridge decks under the 
nonlinear regression model with the different types of structures. It was concluded that all three deterioration curves have approximately the same behavior in the beginning of the service. In other words, the period to drop from a condition rating of 9 to 8 is almost the same. Conversely, a difference between the deterioration curves was noticed within condition ratings from 8 to 4 . The steel structure type showed the highest deterioration rates within this range of condition ratings, while the prestress type was found to have the lowest deterioration rates for the same range of condition ratings (Figure 3). On the other hand, the deterioration curves of the concrete structure type are between the steel and prestress type, but closer to the prestress than the steel type. For example, it took around 15, 18, and 20 years to deteriorate from condition rating 9 to 7 for steel, concrete, and prestress structure types, respectively. Furthermore, the periods to drop from a condition rating of 9 to 4 were 40 years for the steel type, while the period was 42 years for both concrete and prestress. All of the deterioration curves became close to each other when they arrived at the condition rating of 3. For example, steel and prestress structure types dropped to condition rating of 3 after 45 years in service, while for concrete structure type, it took 46 years to become a condition rating of 3 .

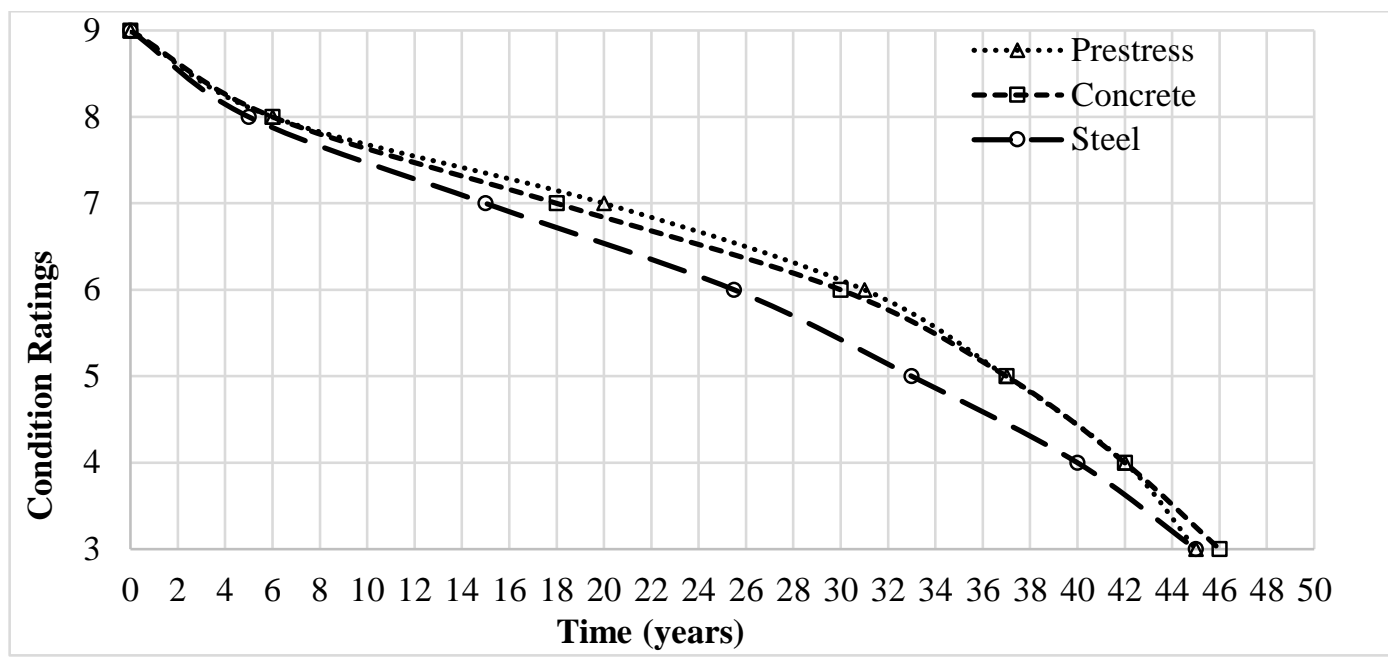

Fig.3. Deterioration Curves of Concrete Bridge Decks with Different Types of Main Structures, ADT $\leq 4,000$, and $\mathrm{SA} \leq 30^{\circ}$.

\subsection{The ADT factor}

The deck is typically the first bridge element that is exposed to deterioration, making ADT a significant factor for consideration [28]. Studying the effect of this factor on bridge deck deterioration improves understanding of the bridge behavior. The ADT factor had a significant effect on the condition of bridge decks as shown in Figure 4. All curves show that increasing the value of ADT leads to increasing the deterioration of the deck condition. Figure 4 shows the deterioration curves of Michigan concrete bridge with two ranges of ADT, different structure type, and skew less than $30^{\circ}$ (i.e., ADT less and more than 4,000 vehicle/day). The bridge decks that have ADT of more than 4,000 (vehicles/day) deteriorated faster than of those decks with ADT less than 4,000 with the same type of structure and skew angle. For example, it took around 18 years to deteriorate from a condition rating of 9 to 7 for bridge decks with ADT less than 4,000 vehicles/day for a, concrete structure and skew angle less than $30^{\circ}$, while it was just about 12 years to drop from a condition rating of 9 to 7 for bridge decks under ADT of more than 4,000 vehicles/day for the same other factors. On the other hand, the deterioration curves became close to each other at the poor conditions (i.e., 4 or 3), under the effect of different ranges of ADT. For example, the periods of time to deteriorate from a condition rating of 9 to 4 were about 40 and 42 years for ADT more and less than 4,000 vehicles/day, respectively. The same conclusion of bridge deck behavior can be seen in the other curves of the same figure.

\subsection{The skew angle factor}

The effect of skew angles on the deterioration rates of bridge decks is similar to the effect of ADT. Increasing the values of skew angles resulted in a significant effect on the deterioration of the bridge decks for all condition ratings from 9 to 4 as shown in Figure 5. For example, bridge decks with skew angles less than $30^{\circ}$ that have concrete structure type and ADT less than or equal to 4,000 vehicles/day can take about 30 years to deteriorate from a condition rating of 9 to 6 , while those with skew angles more than $30^{\circ}$ can take around 24 years to drop from condition ratings of 9 to 6 . Additionally, the same process of deterioration can be noticed for the same factor with different types of main structure (i.e., steel and prestress) in the other curves of the same figure. Furthermore, it has been shown that most Michigan concrete bridge decks could take at least 40 years before arriving to the 
condition rating of 3 , which was the critical rating of most types of bridges (i.e., time needed for major rehabilitation, and/or replacement).

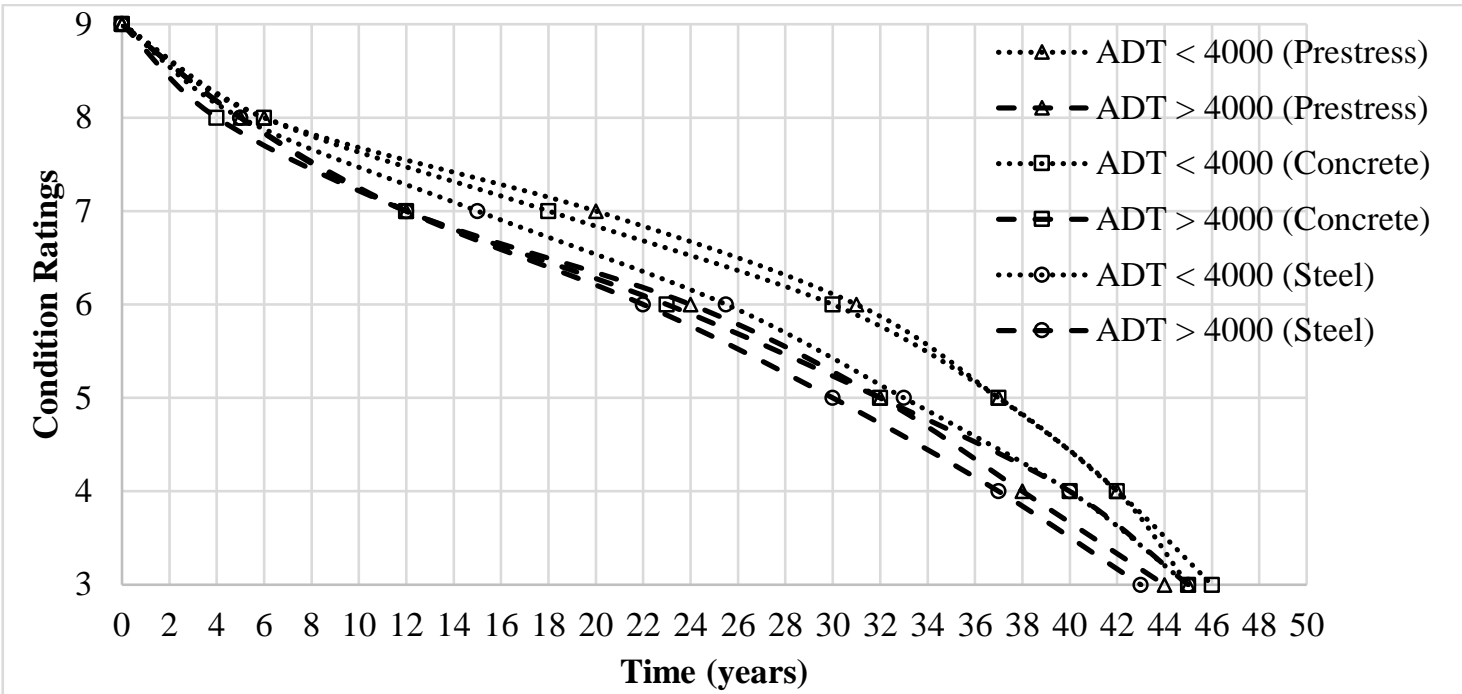

Fig. 4. Deterioration Curves of Concrete Bridge Decks with Different Ranges of ADT, Main Structure Type, and Skew $<30^{\circ}$.

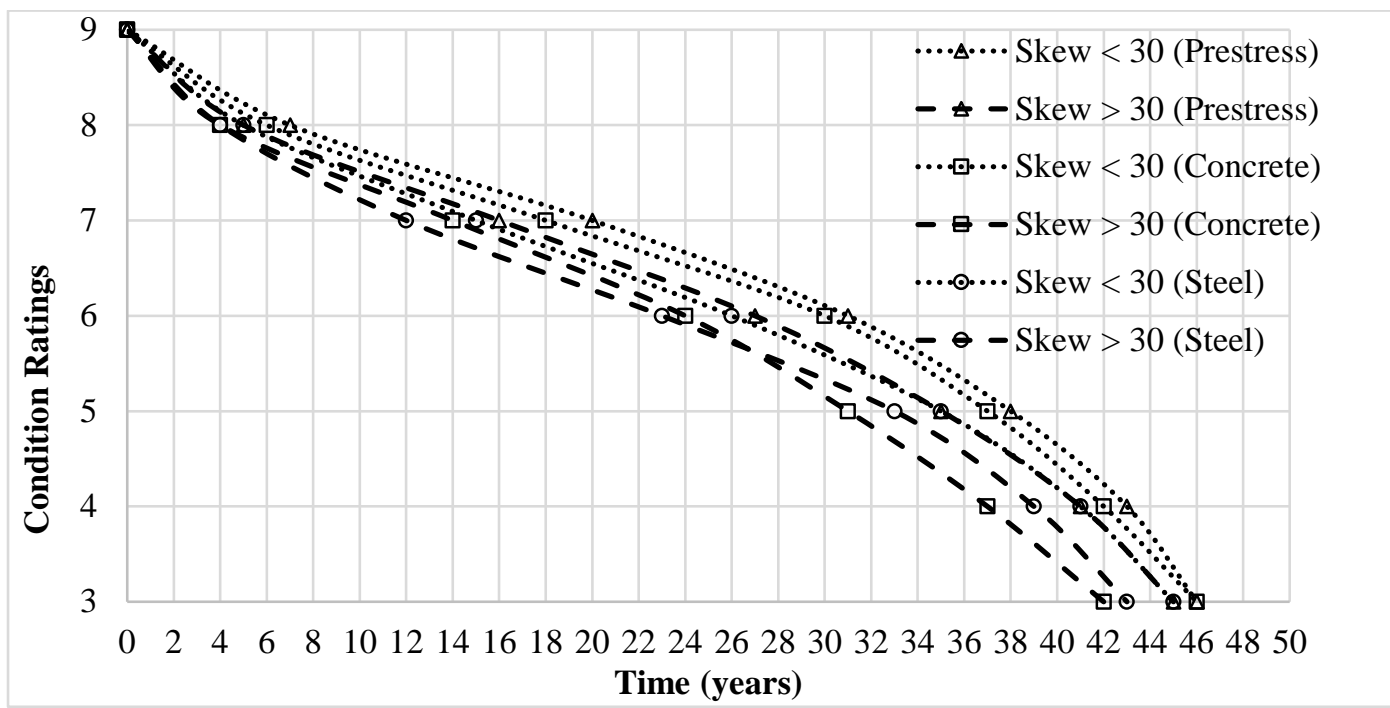

Fig. 5 Deterioration Curves of Concrete Bridge Decks with Different Ranges of Skew, Main Structure Type, and ADT < 40,000.

\section{Concluding remarks}

Michigan concrete bridge decks condition ratings during the period from 1992 to 2016 were analyzed and used to develop statistical, Markov chain, and nonlinear regression models to optimally evaluate the deterioration rates and to investigate the impact of ADT, main structure types, and skew angles on the deck condition. According to the literature, the Markov chain model is considered the most commonly used method for evaluating the deterioration rates of bridges. This pilot study has also found that the lognormal statistical model is comparable to the Markov chain approach and has sought to further optimize the analysis and prediction by developing a nonlinear regression model that is based on the lognormal and Markov chain approaches.

This paper illustrated that the deterioration rates of Michigan concrete bridge decks are fast at the beginning and end of their service lives, and slow in the middle. Additionally, they can take at least 40 years before dropping to condition rating of 3, which was the indicator for serious repair, or reconstruction, regardless of the amount of ADT, main structure types, and skew angles of bridges. Consequently, inspection schedules and maintenance actions for Michigan concrete bridge decks can be planned based on their deterioration rates under the effect of the factors discussed in this paper. 
The analysis and modeling approaches presented in this paper provide a framework for further investigations into the best prediction modeling and analysis of all the factors that impact bridge deck performance. Each state would need to develop its own models based on its specific data. Furthermore, more research studies will be required to assess each of the factors in more details. Such studies would include more ranges for ADT and skew angles to provide a detailed impact analysis of the deterioration rates of concrete bridge decks.

\section{References}

[1] Wang YZ, He TQ, Liu S, Sun YY. Condition assessment for existing reinforced concrete bridges. In: Earth \& Space 2008: Engineering, Science, Construction, and Operations in Challenging Environments; 2008. p. $1-8$.

[2] Madanat S, Mishalani R, Ibrahim WH. Estimation of infrastructure transition probabilities from condition rating data. Journal of Infrastructure Systems. 1995;1(2):120-125.

[3] Mašović S, Hajdin R. Modelling of bridge elements deterioration for Serbian bridge inventory. Structure and Infrastructure Engineering. 2014;10(8):976-987.

[4] Morcous G, Lounis Z, Cho Y. An integrated system for bridge management using probabilistic and mechanistic deterioration models: Application to bridge decks. KSCE Journal of Civil Engineering. 2010;14(4):527-537.

[5] Moomen M. Deterioration modeling of highway bridge components using deterministic and stochastic methods. [Master's thesis]. University of Purdue, West Lafayette, Indiana. 2016.

[6] National Bridge Inspection Standards (NBIS). Federal Register. 2004; 69(239): 74419-74439.

[7] Agrawal AK, Kawaguchi A, Chen Z. Deterioration rates of typical bridge elements in New York. Journal of Bridge Engineering. 2010;15(4):419-429.

[8] Hema J, Guthrie WS. Concrete bridge deck condition assessment guidelines. Rep. No. UT-05-01. Utah Dep. of Transportation. 2005.

[9] Sun L, Gu W. Pavement condition assessment using fuzzy logic theory and analytic hierarchy process. Journal of Transportation Engineering. 2011;137(9):648-655.

[10] Bolukbasi M, Mohammadi J, Arditi D. Estimating the future condition of highway bridge components using national bridge inventory data. Practice Periodical on Structural Design and Construction. 2004;9(1):16-25.

[11] Caner A, Yanmaz AM, Yakut A, Avsar O, Yilmaz T. Service life assessment of existing highway bridges with no planned regular inspections. Journal of Performance of Constructed Facilities. 2008;22(2):108-114.

[12] Nasrollahi M, Washer G. Estimating inspection intervals for bridges based on statistical analysis of national bridge inventory data. Journal of Bridge Engineering. 2015;20(9):04014104.

[13] Tabatabai H, Tabatabai M, Lee CW. Reliability of bridge decks in Wisconsin. Journal of Bridge Engineering. 2011;16(1):53-62.

[14] Stacey V. Expected service life of Michigan department of transportation reinforced concrete bridge decks. University of Michigan, Michigan. 2015.

[15] Cremona C, Poulin B. Standard and advanced practices in the assessment of existing bridges. Structure and Infrastructure Engineering. 2017;13(4):428-439.

[16] Omar T, Nehdi ML. Condition assessment of reinforced concrete bridges: Current practice and research challenges. Infrastructures. 2018;3(3):36.

[17] Tolliver D, Lu P. Analysis of bridge deterioration rates: A case study of the northern plains region. Journal of the Transportation Research Forum. 2011;50(2), 87-100.

[18] Federal Highway Administration (FHWA). Recording and coding guide for the structure inventory and appraisal of the nation's bridges. Rep. No. FHWA-PD-96-001. Washington, DC. 1995.

[19] Goodwin BT. Bridge deck condition assessment using destructive and nondestructive methods. [Master's thesis]. Missouri University, Rolla, MO. 2014.

[20] Michigan Department of Transportation (MDOT). National bridge inventory rating scale. Michigan Department of Transportation, MI. 2015.

[21] AASHTO. Manual for bridge element inspection, 1st Ed. American Association of State Highway and Transportation Officials (AASHTO), Washington D.C. 2013.

[22] Sobanjo J, Mtenga P, Rambo-Roddenberry M. Reliability-based modeling of bridge deterioration hazards. Journal of Bridge Engineering. 2010;15(6):671-683.

[23] Mehrannia H, Pakgohar A. Using easy fit software for goodness-of-fit test and data generation. International Journal of Mathematical Archive. 2014; 5(1): 18-124.

[24] Engmann S, Cousineau D. Comparing distributions: the two-sample Anderson-Darling test as an alternative to the Kolmogorov-Smirnoff test. Journal of Applied Quantitative Methods. 2011;6(3):1-17.

[25] Aristizabal RJ. Estimating the parameters of the three-parameter Lognormal distribution. [Master's thesis]. Florida International University, Miami, Florida. 2012. 
[26] Li L, Sun L, Ning G. Deterioration prediction of urban bridges on network level using Markov-chain model. Mathematical Problems in Engineering. 2014;2014.

[27] Fu G, Devaraj D. Methodology of homogeneous and non-homogenous Markov chains for modelling bridge element deterioration (Final report). Michigan department of transportation. Wayne State University. 2008.

[28] Jiang Y, Saito M, Sinha KC. Bridge performance prediction model using the Markov chain. Transportation Research Record. 1988;1180:25-31.

[29] Hatami A, Morcous G. Developing deterioration models for nebraska bridges. Pr. No. SPR-P1(11) M302. University of Nebraska-Lincoln, Omaha, Nebraska 68182-0178. 2011.

(C) 2020 by the author(s). This work is licensed under a Creative Commons Attribution 4.0 International License (http://creativecommons.org/licenses/by/4.0/). Authors retain copyright of their work, with first publication rights granted to Tech Reviews Ltd. 